\title{
TRIVIAL STABLE STRUCTURES WITH NON-TRIVIAL REDUCTS
}

\author{
DAVID M. EVANS
}

\begin{abstract}
We offer a new viewpoint on some of the generic structures constructed using Hrushovski's predimensions and show that they are natural reducts of quite straightforward trivial, one-based stable structures.

2000 Mathematics Subject Classification: 03C45.
\end{abstract}

\section{INTRODUCTION}

In the late 1980's, Ehud Hrushovski invented a remarkable construction which produced examples and counterexamples settling several important problems and conjectures in model theory [10]. There is now strong evidence that this construction has wider significance for model theory, as in many cases the objects which it produces occur 'naturally' elsewhere in mathematics. In particular there are connections between the construction and complex analytic geometry [18, 17, 11, 12]; generic complex plane curves [5]; and sparse random graphs [15, 2].

In this paper we offer an alternative viewpoint on Hrushovski's construction which simplifies several aspects aspects of it. We also believe that this way of viewing the construction provides further evidence (if needed) of its 'naturality.'

In the original construction one has an amalgamation class of (finite) structures and distinguished embeddings, defined by non-negativity of a straightforward function called a predimension. In our approach we will see that this class and its embeddings can be viewed as a 'reduct' of a much simpler class of structures (plus embeddings). Using this we show that the $\omega$-stable ab initio structures produced by the construction are reducts of trivial, 1-based stable structures (Theorem 1.9).

In order to describe a special case (closely related to the example in Section 1 of [7]), we begin with a description of a reasonably natural combinatorial object. By a digraph we mean a set of vertices together with an anti-symmetric, irreflexive binary relation on the vertices (the directed edges). Consider the class $\mathcal{D}$ of digraphs in which every vertex has at most two directed edges coming out of it (i.e. has at most two out-neighbours). In such a digraph, call a subset of vertices closed if any out-neighbour of a vertex in the subset is already in the subset. Using a Fraïssé-style amalgamation argument, it is easy to

Date: 18 October 2004. 
show that there is a unique (up to isomorphism) countable digraph $D \in \mathcal{D}$ with the properties that: $D$ is a union of a chain of finite closed subdigraphs; any finite digraph in $\mathcal{D}$ embeds as a closed subdigraph of $D$; any isomorphism between finite, closed subdigraphs of $D$ extends to an automorphism of $D$. It can be shown that $T h(D)$, the theory of $D$, is stable, one-based and trivial (Theorem 1.9(1)).

Now consider the (undirected) graph $H$ obtained by forgetting the orientation on the edges of $D$. By Theorem 1.9(2), $H$ is isomorphic to the 'ab initio' Hrushovski structure constructed using the predimension 'twice number of vertices minus number of edges' on finite graphs. So $H$ is $\omega$-stable of Morley rank $\omega .2$ and it is neither trivial nor one-based (cf. Theorem 1.1).

We can do this more generally and obtain any of the basic ab initio Hrushovski structures produced by an integer-valued predimension as a reduct of a 'natural' stable, trivial one-based structure. This is the content of Section 1 of the paper. Although we have not been able to do the same for the Hrushovski structures of finite Morley rank, we believe our viewpoint offers some extra insight into the algebraic amalgamation required for these constructions. This is the content of Section 2. Finally in Section 3 we observe that the digraph $D$ (and therefore the graph $H$ ) is interpretable in a very natural module. We also show that any pseudoplane arising from a non-one-based reduct of a stable one-based theory (with nfcp) has to satisfy a 'positivity of predimension' inequality (Proposition 3.1). Thus we have a case where such a condition arises from some reasonably natural model-theoretic assumptions.

\section{The AB INITIO CASE}

Suppose $r \geq 2$ and $m, n \geq 1$ are fixed integers. We work with the class $\mathcal{C}$ of finite $r$-uniform hypergraphs, which we regard as structures in a language with a single $r$-ary relation symbol $R\left(x_{1}, \ldots, x_{r}\right)$ whose interpretation is invariant under permutation of coordinates and satisfies $R\left(x_{1}, \ldots, x_{r}\right) \rightarrow \bigwedge_{i<j}\left(x_{i} \neq x_{j}\right)$. If $B \in \mathcal{C}$ consider the predimension

$$
\delta(B)=n|B|-m|R[B]|
$$

where $R[B]$ denotes the set of hyperedges on $B$ (i.e $\left\{\left\{b_{1}, \ldots, b_{r}\right\}: B \mid=\right.$ $\left.\left.R\left(b_{1}, \ldots, b_{r}\right)\right\}\right)$. For $A \subseteq B$, we write $A \leq B$ iff for all $A \subseteq B^{\prime} \subseteq B$ we have $\delta(A) \leq \delta\left(B^{\prime}\right)$, and let $\mathcal{C}_{0}=\{B \in \mathcal{C}: \emptyset \leq B\}$.

We let $\overline{\mathcal{C}}_{0}$ be the set of structures all of whose finite substructure are in $\mathcal{C}_{0}$. If $A \subseteq B \in \overline{\mathcal{C}}_{0}$ we write $A \leq B$ iff $X \cap A \leq X$ for all finite $X \subseteq B$. (This agrees with what was previously defined on $\mathcal{C}_{0}$.) Then the following is well-known and is sometimes referred to as the $a b$ initio case of the Hrushovski construction:

Theorem 1.1. There is a unique countable $M_{0} \in \overline{\mathcal{C}}_{0}$ having the properties: $M_{0}$ is a union of a chain of finite $\leq$-substructures; if $X \leq M_{0}$ is 
finite and $X \leq A \in \mathcal{C}_{0}$, then there is an embedding $\alpha: A \rightarrow M_{0}$ which is the identity on $X$ and $\alpha(A) \leq M_{0}$. Moreover, Th $\left(M_{0}\right)$ is $\omega$-stable, and $M_{0}$ is $\omega$-saturated.

The structure $M_{0}$ is the generic structure for the class $\left(\mathcal{C}_{0}, \leq\right)$. A proof of the above statement can be found in ([3], Theorem 3.34) or [16]. For 'most' choices of $m, n, r$, the theory $T h\left(M_{0}\right)$ is neither trivial, nor one-based: indeed the point of Hrushovski's construction in [10] is to produce regular types whose geometries are not modular. We will show that $M_{0}$ is, however, a reduct of a stable, trivial one-based theory by deriving the amalgamation class $\left(\mathcal{C}_{0}, \leq\right)$ as a 'reduct' of a less complicated one $(\mathcal{G}, \sqsubseteq)$.

The main idea is a connection between the condition $\emptyset \leq B$ and Philip Hall's Marriage Theorem (see Lemma 1.5). A similar observation was exploited in a different way in [5], where it is attributed to Frank Wagner.

The following definition looks technical: in fact it is a way of making sense of the notion of 'directed hypergraph' in a way which is suitable for our context. The remarks following the definition should help explain it and make it look less contrived.

Definition 1.2. Suppose $A$ is an $r$-uniform hypergraph. An $(m, n)$ orientation of $A$ is an integer-valued function $w(x, e)$ defined on pairs $\{(x, e): x \in A, e \in R[A], x \in e\}$ and satisfying:

(1) $0 \leq w(x, e) \leq m$;

(2) for $e \in R[A]$ we have $\sum_{x \in e} w(x, e)=m$;

(3) for $x \in A$ we have $\sum_{x \in e} w(x, e) \leq n$.

We refer to the pair $(A, w)$ as an $(m, n)$-oriented hypergraph and denote the class of these by $\overline{\mathcal{G}}$, and the finite members by $\mathcal{G}$.

Remarks 1.3. As $m, n, r$ are fixed for our purposes, we shall henceforth omit them from the terminology. It should be clear what we mean by a substructure of an oriented hypergraph: we restrict $R$ and the function $w$. It should also be clear that as $w$ can take only finitely many values, we can regard oriented hypergraphs as relational structures (in a richer language).

As a special case, consider what the definition means if $r=2$ (so we are dealing with ordinary graphs) and $m=1$. Thus, by (1) and (2), w selects one vertex from each edge. Think of the edge as being directed away from the selected vertex. Then (3) says that each vertex has at most $n$ directed edges coming out of it. More generally, if $r$ is arbitrary but $m=1$ still, then $w$ distinguishes one vertex from each hyperedge and any vertex is the distinguished vertex of at most $n$ hyperedges.

Definition 1.4. If $(B, w)$ is an oriented hypergraph and $A \subseteq B$ we write $A \sqsubseteq B$ to mean that if $a \in A$ and $w(a, e)>0$, then $e \subseteq A$. 
So for example, if we are in the case $r=2, m=1$, then $A \sqsubseteq B$ means that no directed edge points out of $A$. In general, there is a closure operation associated with $\sqsubseteq$. If $(B, w) \in \overline{\mathcal{G}}$ and $X \subseteq B$, then define $\mathrm{cl}_{B}^{\prime}(X)$ to be the closure of $X$ under the operation of adjoining to $X$ all vertices in edges $e$ with $w(x, e)>0$ for some $x \in e \cap X$. It is clear that this is a closure operation and $\mathrm{cl}_{B}^{\prime}(X)$ is the smallest subset $A$ with $X \subseteq A \sqsubseteq B$. Note that this closure operation is disintegrated: $\operatorname{cl}_{B}^{\prime}(X)=\bigcup_{x \in X} \operatorname{cl}_{B}^{\prime}(x)$.

The main observation comnnecting the classes $\left(\mathcal{C}_{0}, \leq\right)$ and $(\mathcal{G}, \sqsubseteq)$ is the following:

Lemma 1.5. Suppose $B$ is a finite $r$-uniform hypergraph and $A \subseteq B$. Then $\emptyset \leq A \leq B$ iff there is an orientation $(B, w)$ of $B$ in which $A$ is a closed subset. In particular, $B \in \mathcal{C}_{0}$ iff there is an orientation of $B$.

Proof. $(\Leftarrow:)$ Suppose there is an orientation $(B, w)$ in which $A$ is closed. By considering substructures, and the special case where $A=\emptyset$, it suffices to show that $\delta(B) \geq \delta(A)$, i.e. $n|B \backslash A| \geq m|R[B] \backslash R[A]|$.

Compute the $\operatorname{sum} \sum\{w(x, e): x \in B \backslash A, x \in e \in R[B]\}$ in two different ways. Firstly, it is $\leq n|B \backslash A|$. Secondly, because $A \sqsubseteq B$ the edges making a non-zero contribution to the sum are those in $R[B] \backslash$ $R[A]$. Also, if $e \in R[B] \backslash R[A]$ and $w(x, e)>0$, then $x \in B \backslash A$. So the sum is equal to $m|R[B] \backslash R[A]|$. This gives the desired inequality.

$(\Rightarrow:)$ The idea is to interpret the condition $A \leq B$ as a condition on a certain bipartite graph which will allow us to use Hall's Marriage Theorem (cf. [4], Theorem III.3.7) to obtain a complete matching on the bipartite graph: the orientation will then be derived from the matching. Indeed, Hall's theorem applies directly in the case where $m=n=1, A=\emptyset$ and the bipartite graph is taken as the incidence graph between hyperedges and vertices: the matching picks out the vertex of the hyperedge which is to be distinguished. The reader will probably have little difficulty adapting this argument to prove the general case, but we give a slightly different style of argument in terms of flows in directed graphs. For this, we shall use the terminology of ([4], Section III.1), but for the convenience of the reader we repeat some of it here.

Suppose we have a finite digraph $\Gamma$ with two distinguished vertices $s$ (the source) and $t$ (the sink). Suppose also that to each other vertex there is assigned a non-negative real number called its capacity. A flow on $\Gamma$ (with this capacity function) is a function which assigns a non-negative real to each directed edge of $\Gamma$ and has the property that at each vertex $\neq s, t$ the sum of its values on the in-edges is equal to the sum of its values on the out-edges, and this number is at most the capacity of the vertex. The value of the flow is the sum of the values of the flow on the out-edges of $s$ minus the sum of its values on the in-edges at $s$ (and this is equal to the sum of the values of the flow 
on the in-edges of $t$ minus the sum of its values on the out-edges at $t$ ). A (vertex) cut of $\Gamma$ (with the capacity function) is a subset $S$ of the vertices (excluding $s, t$ ) with the property that any flow on $\Gamma \backslash S$ has value zero. The capacity of $S$ is the sum of the capacities of the vertices in $S$.

We now turn to the proof of our result. Note first that, inductively, we may assume that there is an orientation $v$ of $A$. We have to say how to orient the edges $e \in R[B] \backslash R[A]$. Note that to ensure $A \sqsubseteq B$ in the final orientation $w$ we should have $w(a, e)=0$ for any such $e$ and $a \in A$.

Construct a directed graph $\Gamma$ with vertices a source $s$, a sink $t$, and other vertices $V_{1}=R[B] \backslash R[A], V_{2}=B \backslash A$. There are directed edges from $s$ to all vertices in $V_{1}$ and from all vertices in $V_{2}$ to $t$, and from $e \in V_{1}$ to $x \in V_{2}$ whenever $x \in e$ (and these are the only directed edges). We assign the capacity $m$ to all vertices in $V_{1}$ and $n$ to all vertices in $V_{2}$. Consider a flow $f$ of maximum possible value. Recall ([4], Theorem III.1.2) that we may also assume that $f$ is integer valued. Clearly the value of $f$ is at most $m\left|V_{1}\right|$. We make the:

Claim: $f$ has value $m\left|V_{1}\right|$.

Suppose we have proved this. Then the flow out of every vertex of $V_{1}$ is exactly $m$. For $e \in V_{1}$ and $x \in V_{2}$ with $x \in e$, define $w(x, e)=f(e, x)$, the value $f$ assigns to the directed edge from $e$ to $x$. Extend this by setting $w(x, e)=0$ for $e \in V_{1}, x \in e \cap A$ and $w(x, e)=v(x, e)$ for $e \in R[A], x \in e$. Then $w$ is an orientation of $B$ in which $A$ is a closed subset, as required.

So it remains to prove the claim. Suppose $f$ has value $<m\left|V_{1}\right|$. By the max-flow, min-cut theorem of Ford and Fulkerson ([4], Theorem III.1.4), there is a cut $S$ with the value of $f$ as its capacity. Let $S_{i}=$ $S \cap V_{i}$. So we have

$$
m\left|S_{1}\right|+n\left|S_{2}\right|<m\left|V_{1}\right|
$$

Thus

$$
\left|S_{2}\right|<\frac{m}{n}\left(|R[B]|-|R[A]|-\left|S_{1}\right|\right) .
$$

Now, there are no directed edges between $V_{1} \backslash S$ and $V_{2} \backslash S$ : otherwise we could use such an edge to produce a flow on $\Gamma \backslash S$ with non-zero value, contradicting that $S$ is a cut. So all the directed edges out of $V_{1} \backslash S$ end in $S_{2}$. Let $X$ be the union of the set of hyperedges $V_{1} \backslash S$. It follows that $|X \backslash A| \leq\left|S_{2}\right|$, i.e.

$$
|X|-|X \cap A| \leq\left|S_{2}\right| \text {. }
$$

On the other hand,

$$
|R[X] \backslash R[A]| \geq\left|V_{1} \backslash S\right|=|R[B]|-|R[A]|-\left|S_{1}\right| .
$$

Putting these inequalities together we obtain

$$
|X|-|X \cap A|<\frac{m}{n}(|R[X]|-|R[X \cap A]|) .
$$


In other words $\delta(X \cap A)>\delta(X)$. But as $A \leq B$ we have $A \cap X \leq X$ : a contradiction. This establishes the claim, and finishes the proof of the lemma.

Remarks 1.6. If $m, n$ are not integers (but still $>0$ ) then the proof shows that the lemma still holds as long as we drop the requirement that the orientation be integer valued.

Lemma 1.7. Suppose $A \subseteq B \in \mathcal{C}_{0}$. If $w$ is an orientation of $B$ in which $A$ is closed and $v$ is any orientation of $A$, then $w^{\prime}$ defined by $w^{\prime}(x, e)=v(x, e)$ if $e \subseteq A$, and $w^{\prime}(x, e)=w(x, e)$ if $e \nsubseteq A$, is an orientation of $B$ (in which $A$ is closed). In particular, if $A \leq B$, then any orientation of $A$ can be extended to an orientation of $B$ in which $A$ is closed.

Proof. For the first statement, one just checks the definitions. The second statement then follows as by Lemma 1.5, if $A \leq B$ then there is some orientation of $B$ in which $A$ is closed (in fact the proof of Lemma 1.5 shows that any orientation of $A$ extends to an orientation of $B$ in which $A$ is closed).

Lemma 1.8. (Full Amalgamation) If $A$ is a common substructure of oriented hypergraphs $(B, w)$ and $(C, u)$ and $A$ is closed in $(C, u)$, then the free amalgam $(F, v)$ of $(B, w)$ and $(C, u)$ over $A$ is an oriented hypergraph in which $B$ is closed.

Proof. Let us be clear about what $(F, v)$ is. The domain $F$ is the disjoint union of $B$ and $C$ over $A$; hyperedges on $F$ are precisely the hyperedges of $B$ and those of $C$; the orientation $v$ is then the union of $w$ and $u$ (the assumption that $A$ is a common substructure means that these agree on point-hyperedge pairs in $A$ ).

First we show $B \sqsubseteq F$. Suppose $b \in B, e$ is an edge of $F$ not contained in $B$ and $v(b, e)>0$. Then $e$ is an edge of $C$, so $b \in C \cap B=A$. But as $A \sqsubseteq C$ this implies $e \subseteq A \subseteq B$ : a contradiction. Thus $B \sqsubseteq F$.

To check that $v$ is an orientation of $F$ we clearly only have to check Definition 1.2 (3). If $x \in B \backslash C$ and $x \in e \in R[F]$ then $e \subseteq B$, so $\sum_{e: x \in e} v(x, e) \leq n$. Likewise if $x \in C \backslash B$. So suppose $x \in A, e \in R[F]$ and $v(x, e)>0$. If $e \subseteq C$ then (as $A \sqsubseteq C$ ) we have $e \subseteq A$. Thus in any case $e$ is contained in $B$, and we get condition 1.2 (3) by the corresponding condition in $(B, w)$.

Thus we have an amalgamation class $(\mathcal{G}, \sqsubseteq)$. It is clear that $\mathcal{G}$ has only finitely many isomorphism types of each finite size (in fact we can think of the structures as being in a finite language), thus, by the usual Fraïssé-style construction there is a unique countable generic structure $N_{0}$ for $(\mathcal{G}, \sqsubseteq)$. Recall that $M_{0}$ denotes the countable generic for $\left(\mathcal{C}_{0}, \leq\right)$ (Theorem 1.1). Our main theorem is part (2) of the following.

Theorem 1.9. With the above notation: 
(1) $N_{0}$ is stable, one-based and trivial.

(2) The reduct of $N_{0}$ to the hypergraph language is isomorphic to $M_{0}$.

Proof. (1) We follow, omitting some details, the development in [7] (see especially Lemma 1.2 there). First, we axiomatize $T h\left(N_{0}\right)$ by the following set $T^{\prime}$ of sentences. We have the axioms describing the class $\overline{\mathcal{G}}$ (in whatever language we have chosen). To these we add axioms of the form:

$$
\forall \bar{x} \exists \bar{y}\left(\Delta_{X}(\bar{x}) \rightarrow \Delta_{X, A}(\bar{x}, \bar{y}) \wedge \chi(\bar{x} \bar{y})\right)
$$

where $X \sqsubseteq A \in \mathcal{G}, \Delta_{X}, \Delta_{X, A}$ denote the basic diagrams of $X, A$ with the variables $\bar{x}$ representing $X$ and the variables $\bar{y}$ representing $A \backslash X$. The formula $\chi(\bar{x} \bar{y})$ expresses that 'if $y \in \bar{y}$ and $w(y, e)>0$ then $e \subseteq \bar{x} \cup \bar{y}$.' Thus if $D \in \overline{\mathcal{G}}$ and $D=\chi(\bar{a} \bar{b})$, then $\operatorname{cl}_{D}^{\prime}(\bar{a} \bar{b})=\operatorname{cl}_{D}^{\prime}(\bar{a}) \cup \bar{b}$. Consistency of $T^{\prime}$ follows from full amalgamation. In fact $N_{0}$ is a model of $T^{\prime}$ (build a countable model of $T^{\prime}$ as a union of a chain of finite structures each closed in the next: the result must also satisy the characteristic properties of the generic $N_{0}$ ).

Note that in an $\omega$-saturated model $N$ of $T^{\prime}$, if $X \sqsubseteq N$ is the closure of a finite set and $X \sqsubseteq A \in \overline{\mathcal{G}}$ where $A$ is also the closure of a finite set, then there is an embedding over $X$ of $A$ into $N$ with closed image. This allows us to show, by a back-and-forth argument, that $T^{\prime}$ is complete and types are described by quantifier-free types of closures. Counting types gives stability, and (arguing as for Lemma 1.2 of [7]), in a saturated model, two small closed subsets are independent over their intersection. From this, and the fact that closure is disintegrated, we obtain that $T^{\prime}$ is one-based and trivial. This gives (1).

(2) It is enough to show that the (unoriented) reduct $M$ satisfies the characteristic properties of the generic given in Theorem 1.1. As $N_{0}$ is a union of a chain of finite $\sqsubseteq$-substructures, we have that $M$ is a union of a chain of finite $\leq$-substructures (by Lemma 1.5).

Let $X \leq M$ be finite and $X \leq A \in \mathcal{C}_{0}$. Let $Y=\operatorname{cl}_{N_{0}}^{\prime}(X)$. Note that $Y$ is finite (as $N_{0}$ is a union of a chain of finite $\sqsubseteq$-substructures). The orientation of $X$ as a substructure of $N_{0}$ extends to an orientation $w$ of $A$ (Lemma 1.7). Let $F$ be the free amalgam of $(A, w)$ and $Y$ over $X$, as in Lemma 1.8. As $X \sqsubseteq(A, w)$ we have $Y \sqsubseteq F$. Also $Y \sqsubseteq N_{0}$, so (using $T^{\prime}$ ) there is an embedding of $F$ into $N_{0}$ which is the identity on $Y$ and has closed image in $N_{0}$. Thus we may assume that $F \sqsubseteq N_{0}$. Now consider this in the reduct $M$. It is clear that (the unoriented) $F$ is the free amalgam in $\mathcal{C}_{0}$ of $A$ and $Y$ over $X$. As $X \leq Y$, it follows that $A \leq F$. But also $F \leq M$, so $A \leq M$, as required.

\section{Finite RANK StRUCTURES}

It is well-known that if a complete theory is stable and one-based (respectively, trivial) and all its types have finite $U$-rank, then any reduct 
is also one-based (respectively, trivial): see ([13], Proposition 4.6.3) and [8]. However, it appears to be an open question as to whether a finite $U$-rank reduct of a stable one-based (trivial) theory is necessarily one-based. In particular, it would be interesting to know whether Hrushovski's strongly minimal set from [10] can be obtained as a reduct of a stable, one-based trivial structure. In this section we try to repeat the construction of the previous section in order to do this. We do not succeed. Nevertheless, this viewpoint gives a different insight into the main amalgamation lemma required for Hrushovski's construction and so is still of interest.

We continue to use the notation of Section 1. The following is one of the key definitions from [10]. Suppose $X \subset Y \in \mathcal{C}_{0}$. We say that the extension $X \subset Y$ is simply algebraic if $\delta(X)=\delta(Y)$ and whenever $X \subset X_{1} \subset Y$, then $\delta(X)<\delta\left(X_{1}\right)$. So $X \leq Y$, but $X_{1} \not \leq Y$ for all $X \subset X_{1} \subset Y$. The extension is minimally simply algebraic if the extension $X_{0} \subset X_{0} \cup(Y \backslash X)$ is not simply algebraic for all proper subsets $X_{0}$ of $X$. We now formulate this in terms of orientations. The following terminology will be convenient.

Definition 2.1. Suppose $(Y, w) \in \mathcal{G}$ and $X \subset Y$. We say that $y \in Y$ is full in $(Y, w)$ if $\sum\{w(y, e): e \in R[Y]\}=n$. We say that $y$ is full in $Y$ if the orientation $w$ is clear from the context.

Lemma 2.2. Suppose $X \subset Y \in \mathcal{C}_{0}$. Then the following are equivalent.

(1) $X \subset Y$ is simply algebraic.

(2) $X \leq Y$, and for every orientation $w$ of $Y$ in which $X$ is closed, $y$ is full in $(Y, w)$ and $X \cup \operatorname{cl}_{(Y, w)}^{\prime}(y)=Y$, for all $y \in Y \backslash X$.

(3) There is an orientation $w$ of $Y$ in which $X$ is closed, and $y$ is full in $(Y, w)$ and $X \cup \operatorname{cl}_{(Y, w)}^{\prime}(y)=Y$, for all $y \in Y \backslash X$.

Proof. First we show $(1 \Rightarrow 2)$. Take an orientation $w$ of $Y$ in which $X$ is closed. It follows that

$$
\begin{gathered}
n|Y \backslash X| \geq \sum_{y \in Y \backslash X}\{w(y, e): y \in e\}= \\
=\sum_{e \in R[Y] \backslash R[X]}\{w(y, e): y \in Y \backslash X\}=m|R[Y] \backslash R[X]| .
\end{gathered}
$$

As $\delta(Y)=\delta(X)$ we have equality here and so obtain the fullness condition in (2). For the other condition, let $X_{1}=X \cup \mathrm{cl}_{(Y, w)}^{\prime}(y)$. Then $X \subset X_{1} \sqsubseteq(Y, w)$, so $X_{1} \leq Y$, and therefore $X_{1}=Y$.

The implication $(2 \Rightarrow 3)$ is clear, so now we show $(3 \Longrightarrow 1)$. The counting in the first part of the proof shows that $\delta(Y)=\delta(X)$. On the other hand, if $X \subset X_{1} \subset Y$ then $X_{1} \nsubseteq(Y, w)$, so there is $x \in X_{1} \backslash X$ and an edge $e$ of $Y$ with $w(x, e)>0$ and $e$ not contained in $X_{1}$. So $e$ is not an edge in the substructure on $X_{1}$, and therefore $\sum\left\{w(x, f): f \in R\left[X_{1}\right]\right\}<n$. Counting as in the proof of $(1 \Rightarrow 2)$ gives $\delta(X)<\delta\left(X_{1}\right)$. 
Definition 2.3. Suppose $(Y, w) \in \mathcal{G}$ and $X \subset Y$. We say that $X \subset Y$ is a primitive extension if:

(1) $X \sqsubseteq(Y, w)$;

(2) if $y \in Y \backslash X$, then $y$ is full in $Y$ and $\operatorname{cl}_{(Y, w)}^{\prime}(y)=Y$;

(3) $Y=\bigcup\{e: e \in R[Y] \backslash R[X]\}$.

Corollary 2.4. Suppose $X \leq Y \in \mathcal{C}_{0}$. Then the following are equivalent.

(1) $X \subset Y$ is minimally simply algebraic;

(2) there is an orientation $w$ of $Y$ in which $X \subset(Y, w)$ is a primitive extension;

(3) for every orientation $w$ of $Y$ in which $X$ is closed, the extension $X \subseteq(Y, w)$ is primitive.

Proof. By the previous lemma, the issue is the minimality. To see that $(2 \Rightarrow 1)$, suppose we have an orientation in which $X \subset Y$ is a primitive extension. If we delete some points of $X$ then we destroy some edge which is not entirely contained in $X$, and therefore some point of $Y \backslash X$ ceases to be full. Thus $X \leq Y$ is minimally simply algebraic.

For $(1 \Rightarrow 3)$, suppose $X \leq Y$ is minimally simply algebraic and $w$ is any orientation of $Y$ in which $X$ is closed. So (1), (2) in the above definition hold. If $x \in X$ is not in an edge containing some point of $Y \backslash X$, then we can delete it (and all edges containing it) and still have conditions (1), (2) of the definition above holding. Thus by the lemma, $X \backslash\{x\} \subset Y \backslash\{x\}$ is simply algebraic: a contradiction.

Remarks 2.5. If $X \leq Y$ is minimally simply algebraic, there can be orientations of $Y$ in which $X$ is not closed. The easiest example is with $m=n=1$ and a ternary relation. Take $Y$ to be three points forming a single edge, and $X$ two of the points. If we orient $Y$ so that one of the points of $X$ is the distinguished vertex, then of course $X$ is not closed in $Y$ in this orientation.

Lemma 2.6. If $(A, w) \in \overline{\mathcal{G}}$, and $X \subseteq Y_{1}, Y_{2} \subseteq A$, where $X \subseteq Y_{i}$ is a primitive extension, then either $Y_{1}=Y_{2}$ or $Y_{1} \cap Y_{2}=X$. Moreover $\mathrm{cl}_{A}^{\prime}\left(Y_{1}\right)=Y_{1} \cup \mathrm{cl}_{A}^{\prime}(X)$.

Proof. For the first part, suppose $y \in Y_{1} \cap Y_{2} \backslash X$. As $y$ is full in both $Y_{1}$ and $Y_{2}$, any edge $e$ of $A$ with $w(y, e)>0$ is contained in $Y_{1} \cap Y_{2}$. The claim then follows from the definition of primitivity. The second part follows from fullness in $Y_{1}$ of points in $Y_{1} \backslash X$.

The difference between the $a b$ initio version of Hrushovski's construction in Section 1, and the strongly minimal set construction in [10] is that in the former, types corresponding to minimally simply algebraic extensions are minimal types, whereas in the latter, these are 'collapsed' to algebraic types. 
We can do the same thing using oriented structures and primitive extensions. To construct the strongly minimal set one knows, for model theoretic reasons, that the multiplicities $\mu(Y, X)$ of the minimally simply algebraic extensions $X \leq Y$ cannot be specified completely arbitrarily. However, the combinatorial reasons for this are somewhat mysterious. In [10] Hrushovski works with a lower bound $\mu(Y, X) \geq \delta(X)$ and raises the question as to what the precise lower bound is.

in contrast, it can be shown that the class of finite oriented structures with the multiplicities of primitive extensions spcified arbitrarily is an $\sqsubseteq$-amalgamation class. Thus one can form a corresponding oriented generic structure. Of course, when one passes to the unoriented reduct, different primitive extensions can give rise to isomorphic minimally simply algebraic extensions and thereby higher multiplicities appear in the reduct. But the real issue here is that the reduct is not $\leq$-homogeneous. The problem is that Lemma 1.7 fails in the situation where multiplicities of primitive extensions are restricted: in the restricted class, an orientation of a $\leq$-substructure need not extend to an orientation of the larger structure (in the same class).

We take a different approach which avoids this problem, but the amalgamation lemma will fail for the class of oriented structures we consider. Nevertheless, this approach will give a different proof of Hrushovski's algebraic amalgamation lemma for the unoriented structures.

Suppose, as in [10], that for each minimally simply algebraic extension $X \subset Y$ in $\mathcal{C}_{0}$ we have a natural number $\mu(Y, X)$, and let $\mathcal{C}^{\mu}$ be the class of structures in $\mathcal{C}_{0}$ which omit substructures consisting of $\mu(Y, X)+1$ copies of an m.s.a. extension $Y \supset X$ over a fixed $X$.

Let $\mathcal{G}^{\mu}$ consist of all orientations of elements of $\mathcal{C}^{\mu}$. It is clear that Lemma 1.7 holds for $\mathcal{C}^{\mu}$ and orientations in $\mathcal{G}^{\mu}$. On the other hand, $\mathcal{G}^{\mu}$ is usually not an amalgamation class. Take some m.s.a. extension $X \subset Y$ which admits two non-isomorphic orientations $X \subset\left(Y_{1}, w_{1}\right)$ and $X \subset\left(Y_{2}, w_{2}\right)$ agreeing on $X$. For $i=1,2$, let $B_{i} \in \mathcal{G}^{\mu}$ consist of $\mu(Y, X)-1$ copies of $\left(Y_{1}, w_{1}\right)$ over $X$ (- call this $\left.A\right)$ together with a copy of $\left(Y_{i}, w_{i}\right)$. Then we cannot amalgamate $B_{1}$ and $B_{2}$ over $A$ in $\mathcal{G}^{\mu}$.

The following gives a different interpretation of Hrushovski's lower bound for $\mu$.

Lemma 2.7. Suppose $X \subseteq Y$ is a m.s.a. extension in $\mathcal{C}_{0}$. Suppose $A$ is the disjoint union over $X$ of $r$ copies $Y_{1}, \ldots, Y_{r}$ of $Y$ and $w$ is an orientation of $A$. If $r>\delta(X)$, then $X \sqsubseteq Y_{i}$ for some $i \leq r$.

Proof. Suppose $X \nsubseteq Y_{i}$ for each $i \leq r$. So there is $y_{i} \in Y_{i} \backslash X, x_{i} \in X$ and $e_{i} \in R\left[Y_{i}\right]$ with $w\left(x_{i}, e_{i}\right) \geq 1$ and $y_{i} \in e_{i}$. As the $Y_{i}$ are pairwise disjoint over $X$, the edges $e_{i}$ are distinct. Now sum $w$ over vertices in $X$. We obtain $r+m|R[X]| \leq n|X|$ and so $r \leq \delta(X)$. 
Corollary 2.8. (Self-sufficient amalgamation: [10], Lemma 4.) Suppose that $\mu(Y, X) \geq \delta(X)$ for all minimally simply algebraic extensions $X \subseteq Y$ in $\mathcal{C}_{0}$. If $B_{1}, B_{2} \in \mathcal{C}^{\mu}$ and $A$ is a common substructure with $A \leq B_{1}, B_{2}$, then $B_{1}, B_{2}$ can be amalgamated over $A$ into some structure $C \in \mathcal{C}^{\mu}$ with $B_{1}, B_{2} \leq C$.

Proof. The proof is by induction on $\left|B_{1} \backslash A\right|+\left|B_{2} \backslash A\right|$. Let $F$ be the free amalgam of $B_{1}$ and $B_{2}$ over $A$ and suppose $F \notin \mathcal{C}^{\mu}$. Take an orientation of $B_{1}$ in which $A$ is closed, and extend the orientation of $A$ to an orientation of $B_{2}$. The result is an orientation $w$ of $F$. By assumption, there exist m.s.a. extensions $X \subseteq Y_{1}, \ldots, Y_{r}$ in $F$, pairwise intersecting in $X$ and isomorphic over $X$, such that $r>\mu\left(Y_{1}, X\right)$.

Claim: $X \subseteq A$.

Once we have this the proof is straightforward. For any $i$ either $Y_{i} \subseteq A$ or $Y_{i} \cap A=X$ (by simple algebraicity). So we can assume (as $\left.B_{1}, B_{2} \in \mathcal{C}^{\mu}\right)$ that $Y_{i} \subseteq B_{i}$ and $Y_{i} \cap A=X$ for $i=1,2$. But then $A \cup Y_{i} \sqsubseteq B_{i}$ (by Lemma 2.6) and $A \cup Y_{i}$ is the free amalgam of $A$ and $Y_{i}$ over $X$ (for $i=1,2$ ). (To see the latter, note that by fullness, if $e \in R\left[B_{i}\right]$ contains $y \in Y_{i} \backslash X$ and $w(y, e)>0$, then $e \subseteq Y_{i}$. On the other hand, if $z \in A \cap e$ and $w(z, e)>0$ then $e \subseteq A$ as $A \sqsubseteq B_{i}$, so $e$ containes no points of $Y_{i} \backslash X$. Thus there can be no hyperedges of $B_{i}$ which contain both a point of $Y_{i} \backslash X$ and a point of $A \backslash X$ : there is no possibility for the orientation of such a hyperedge.) So $A \cup Y_{i} \leq B_{i}$ are isomorphic over $A$ (as unoriented structures, not necessarily as oriented structures), and we finish by the inductive hypothesis.

So now we prove the claim. By Lemma 2.7 and our assumption, there exists some $i \leq r$ with $X \sqsubseteq Y_{i}$ (in the orientation $w$ ), so $X \subset Y_{i}$ is a primitive extension. For a contradiction, we assume (without loss) that $X \nsubseteq B_{1}$. Let $x \in X \backslash B_{1}$. As $X \subseteq Y_{i}$ is primitive there exist a hyperedge $e$ in $Y_{i}$ containing $x$ and $y \in Y_{i} \backslash X$ with $w(y, e)>0$. As $F$ is a free amalgam and $x \in X \backslash B_{1}$ we have $e \subseteq B_{2}$. Furthermore, because $A \sqsubseteq B_{2}$ we must have $y \in B_{2} \backslash A$. It then follows from primitivity that $Y_{i} \sqsubseteq B_{2}$ and $Y_{i} \backslash X \subseteq B_{2} \backslash A$, i.e. $Y_{i} \cap A \subseteq X$.

As $B_{2} \in \mathcal{C}^{\mu}$ there is some $j \leq r$ with $\bar{Y}_{j} \not \subset B_{2}$. Then $Y_{j} \cap B_{2} \leq Y_{j}$ and so by m.s.a. $Y_{j} \cap B_{2}=X$ (as $X \subseteq B_{2}$ ). But $x$ is in a hyperedge with some element of $Y_{j} \backslash X$ (by minimality of $X \subseteq Y_{j}$ ) and this is impossible as $F$ is a free amalgam over $A$.

Remarks 2.9. As can be seen from the proof, the lower bound on $\mu$ is used to force $X$ to be a subset of $A$. For certain m.s.a. extensions a different approach is possible, with a lesser bound. For example, working with $r=2$ (graphs) and $\delta(A)=2|A|-|R[A]|$ we can take $\mu\left(Y_{0}, X_{0}\right)=1, \mu\left(Y_{1}, X_{1}\right)=1$ where $Y_{0}$ is a 3 -cycle and $X_{0}$ consists of 2 vertices and $Y_{1}$ is a path of length 2 with the end vertices forming $X_{1}$. In other words, in $\mathcal{C}^{\mu}$ any two vertices have at most one vertex adjacent to both of them, and in the generic, any two vertices have exactly one 
vertex adjacent to both: this is Baldwin's construction of a projective plane from [1]. All we have to check is that if, in the free amalgam $F$ we have vertices $x_{1}, x_{2}$ with two common neighbours $y_{1}, y_{2}$, then we may assume that $x_{1}, x_{2} \in A$. In fact, we could have that $x_{1} \in B_{1} \backslash A$ and $x_{2} \in B_{2} \backslash A$, but then $y_{1}, y_{2} \in A$ and we can interchange the roles of the $y$ 's and $x$ 's to make the induction go through.

\section{Concluding Remarks}

3.1. Interpretation in a module. Hrushovski pointed out to us (private communication) that the digraph $D$ in the Introduction can be interpreted in a module. Thus its graph reduct, the ab initio Hrushovski structure $H$, is also interpretable in the module. Here we give one way of doing this, keeping the interpretation and module as 'natural' as possible.

Let $k$ be any field and $\Lambda=k\left\langle s_{1}, s_{2}\right\rangle$ the free $k$-algebra on two noncommuting generators $s_{1}, s_{2}$. Then $\Lambda$ is a free ideal ring and so is (left) coherent. By ([6], Theorem 4.8), the theory of $\Lambda$-modules has a model completion $T_{\Lambda}$.

Let $S(x, y)$ be the definable relation

$$
\left.(x \neq y) \wedge\left(\left(s_{1} x=y\right)\right) \vee\left(s_{2} x=y\right)\right) .
$$

If $M \models T_{\Lambda}$, then let $M_{S}$ denote the digraph with the digraph relation given by $S$ on $M$. We claim that $M_{S}$ is elementarily equivalent to $D$.

To see this, we use the axiomatization of $T h(D)$ from the proof of Theorem 1.9 (1). This consists of the universal part of $T h(D)$, and axioms $\Delta_{X, A}$ whenever $X \sqsubseteq A \in \mathcal{D}$. It is clear that any finite subdigraph of $M_{S}$ is in $\mathcal{D}$, so $M_{S}$ satifies the universal axioms in $T h(D)$. Now suppose $X$ is a finite sub-digraph of $M_{S}$ and $X \sqsubseteq A \in \mathcal{D}$. Let $V=\langle A \backslash X\rangle_{k}$ be the $k$-vector space with basis $A \backslash X$. We make $M \oplus V$ into a $\Lambda$-module by defining $f_{i}(a)$ for $a \in A \backslash X$ as follows and extending linearly. If there is a directed edge from $a$ to $b$ in the digraph $A$ then let $f_{i}(a)=b$ (for some $i=1,2$ ); if there are no such $b$, then let $f_{i}(a)=a$. As $M$ is existentially closed, we obtain a copy $A^{\prime}$ of $A$ over $X$ inside $M_{S}$ with the only directed edges leaving $A^{\prime}$ coming from $X$. Thus $M_{S}=\Delta_{X, A}$.

Another way of viewing this is that in $M$ the multiplications by $s_{1}$ and $s_{2}$ are behaving as a 'generic pair' of endomorphisms of an infinite dimensional $k$-vector space. Of course, we can dispense with the abelian group structure and simply regard $s_{1}, s_{2}$ as generic pair of functions on an infinite set. More formally, if we consider the free semigroup $S=\left\langle s_{1}, s_{2}\right\rangle$ on two non-commuting generators, then, by [9], the theory of $S$-systems has a model completion and a trivial modification of the argument in the previous paragraph shows that $T h(D)$ is interpretable in this. 
Similar methods can be used to interpret other oriented structures from Theorem 1.9 in natural modules and $S$-systems (although we may have to increase the number of free generators for the ring or semigroup).

3.2. Sparse pseudoplanes. We prove the following. The terminology is explained after the statement of the result.

Proposition 3.1. Suppose $T$ is a complete, stable theory which is onebased and has nfcp. Then any type definable pseudoplane in $T$ is sparse.

We first recall that a stable theory $T$ has $n f c p$ ('does not have the finite cover property') if the quantifier $\exists^{\infty}$ can be eliminated in $T^{e q}$. If $N_{0}$ is one of the oriented stable structures in Theorem 1.9 and $T^{\prime}=T h\left(N_{0}\right)$, then $T^{\prime}$ is nfcp. One way to see this is to recall that any complete theory of modules has nfcp, and nfcp is preserved under interpretation. Thus as $T^{\prime}$ is interpretable in a theory of modules it has nfcp. A rather more standard approach is to use Théorème 6 of [14] and show that any $\omega_{1}$-saturated model of the theory of pairs of models of $T^{\prime}$ is a belle paire.

Next, suppose $T$ is any complete theory and let $\mathbb{C}$ be a sufficiently saturated model of $T$. A type definable pseudoplane $I \subseteq P_{I} \times L_{I}$ is the set of realisations of a (possibly partial) type $I(x, y)$ of $T^{e q}$ such that for all $a \in P_{I}$ and $b \in L_{I}$ the types $I(a, y)$ and $I(x, b)$ are nonalgebraic and whenever $a \neq a^{\prime} \in P_{I}$ and $b \neq b^{\prime} \in L_{I}$, then the sets $\left\{c: \mathbb{C} \models I(a, c) \wedge I\left(a^{\prime}, c\right)\right\}$ and $\left\{d: \mathbb{C} \models I(d, b) \wedge I\left(d, b^{\prime}\right)\right\}$ are finite. If $I(x, y)$ is a complete type, we refer to this as a complete type definable pseudoplane.

It is well known (cf. [13], Proposition 4.1.7) that a stable theory $T$ is one-based if and only if there is no complete type definable pseudoplane in $T$. Thus if $T$ is one-based and has a reduct $T^{-}$which is not onebased, then there is a type $I(x, y)$ which is complete in the smaller language and which is a pseudoplane. Thus in the larger language, $I(x, y)$ gives a type definable pseudoplane, but none of its completions is a pseudoplane.

Finally, we make the following definition. Suppose $X, Y$ are infinite sets and $R \subseteq X \times Y$ is a binary relation. We say that $R$ is sparse if there exists a real number $n>0$ such that for all finite subsetes $A \subseteq X$ and $B \subseteq Y$ we have

$$
|R \cap(A \times B)| \leq n(|A|+|B|) .
$$

Thus we can view Proposition 3.1 as a weak converse to Theorem 1.9: whereas the theorem says that the pseudoplanes given by Hrushovski's ab initio predimension construction can be interpreted in a stable, 1based theory with nfcp, the proposition says that any pseudoplane interpretable in such a theory satisfies a 'positivity of predimension' inequality. 
Proof of Proposition 3.1. Suppose $I(x, y)$ is a type definable pseudoplane in $T^{e q}$ and $\mathbb{C}$ is a saturated model of $T$. As $T$ is one-based, whenever $\mathbb{C} \models I(a, b)$, then either $a \in \operatorname{acl}(b)$ or $b \in \operatorname{acl}(a)$, as otherwise $\operatorname{tp}(a, b)$ gives a complete type definable pseudoplane in $T$. By the nfcp, there exist formulas $\phi_{j}(x, y)$ and $\psi_{j}(x, y)$ and natural numbers $n_{j}, m_{j}$ (for $j \in J$, some indexing set) such that:

$$
\models\left(\forall y \exists^{\leq n_{j}} x \phi_{j}(x, y)\right) \wedge\left(\forall x \exists^{\leq m_{j}} y \psi_{j}(x, y)\right)
$$

and

$$
\models I(x, y) \rightarrow \bigvee_{j \in J} \phi_{j}(x, y) \vee \psi_{j}(x, y) .
$$

So by compactness, there exist a conjuction $\theta(x, y)$ of formulas in $I(x, y)$, a natural number $n$, and formulas $\phi(x, y), \psi(x, y)$ such that

$$
\vDash \theta(x, y) \rightarrow \phi(x, y) \vee \psi(x, y)
$$

and

$$
\models\left(\forall y \exists^{\leq n} x \phi(x, y)\right) \wedge\left(\forall x \exists^{\leq n} y \psi(x, y)\right) .
$$

It follows that the relation on $\mathbb{C}$ given by $\theta$ is sparse; therefore the same is true for $I$.

\section{REFERENCES}

[1] John T. Baldwin, 'An almost strongly minimal non-desarguesian projective plane', Transactions of the American Math. Soc. 342 (1994), 695-711.

[2] John T. Baldwin and Saharon Shelah, 'Randomness and semigenericity', Transactions of the American Mathematical Soc. 349 (1997), 1359-1376.

[3] John T. Baldwin, Niandong Shi, 'Stable generic structures', Annals of Pure and Applied Logic 79 (1996), 1-35.

[4] Béla Bollobás, Modern Graph Theory, Springer Graduate Texts in Mathematics 184, New York, 1998 (second printing 2002).

[5] Olivier Chapuis, Ehud Hrushovski, Pascal Koiran and Bruno Poizat, 'La limite des théories de courbes génériques', J. Symbolic Logic 57 (2002), 24-34.

[6] Paul Eklof and Gabriel Sabbagh, 'Model-completions and modules', Annals of Mathematical Logic 2 (1971), 251-295.

[7] David M. Evans, 'Ample dividing', J. Symbolic Logic 68 (2003), 1385-1402.

[8] John B. Goode, 'Some trivial considerations', J. Symbolic Logic 56 (1991), 624-631.

[9] Victoria A. R. Gould, 'Model companions of $S$-systems', Quart. J. Math. (Oxford) 38 (1987), 189-211.

[10] Ehud Hrushovski, 'A new strongly minimal set', Annals of Pure and Applied Logic 62 (1993), 147-166.

[11] Pascal Koiran, 'The theory of Liouville functions', J. Symbolic Logic 68 (2003), 353-365.

[12] N. Peatfield and B. Zilber, 'Analytic Zariski structures and the Hrushovski construction', Preprint, University of Oxford, April 2003.

[13] Anand Pillay, Geometric Stability Theory, Oxford Logic Guides 32, Clarendon Press, Oxford, 1996.

[14] Bruno Poizat, 'Paires de structures stables', J. Symbolic Logic 48 (1983), 239249. 
[15] Saharon Shelah and Joel Spencer, 'Zero-one laws for sparse random graphs', Journal of the American Math. Soc. 1 (1988), 97-115.

[16] Frank O. Wagner, 'Relational structures and dimensions', in Automorphisms of First-Order Structures, eds. Richard Kaye and Dugald Macpherson, Oxford Science Publications, Oxford 1994, 153-180.

[17] A. J. Wilkie, 'Liouville functions', to appear in Proceedings of Logic Colloquium 2000, Paris, 2000.

[18] B. Zilber, 'Analytic and pseudo-analytic structures', to appear in Proceedings of Logic Colloquium 2000, Paris, 2000.

School of Mathematics, University of East Anglia, Norwich NR4 7TJ, ENGLAND

E-mail address: d.evans@uea.ac.uk 